\title{
ON THE LAW OF QUADRATIC RECIPROCITY*
}

\section{BY ALBERT WHITEMAN}

The following proof of the law of quadratic reciprocity, which depends upon a modified form of the Gaussian criterion, is believed to be new.

According to the usual form of this criterion, if $p$ is any integer not divisible by the odd prime $q$, then $p$ is a quadratic residue or non-residue of $q$ according as in the series

$$
p, 2 p, 3 p, \cdots,(q-1) p / 2
$$

the number of numbers whose least positive remainders $(\bmod q)$ exceed $q / 2$ is even or odd. But, if $\lambda p=\mu q+r, q / 2<r<q$, then $2 \lambda p=(2 \mu+1) q+2 r-q$, and conversely. Hence we have the transformed criterion : $p$ is a quadratic residue or non-residue of $q$ according as the number of least positive odd remainders in the series:

$$
2 p, 4 p, 6 p, \cdots,(q-1) p
$$

is even or odd. $\dagger$

In the following discussion $p, q$ represent any two odd primes such that $q>p$. Let $r$ denote any odd remainder of (1) such that $p<r<q$. Then, for a suitable $\lambda,(1 \leqq \lambda \leqq(q-1) / 2)$,

$$
2 \lambda p \equiv r \quad(\bmod q),
$$

whence

$$
(q+1-2 \lambda) p \equiv p+q-r \quad(\bmod q),
$$

where $p<p+q-r<q$.

Congruences (2) and (3) are identical only for $2 \lambda=(q+1) / 2$, $r=(p+q) / 2$. Hence the odd remainders of (1) that are greater than $p$ may be arranged in pairs by means of (2) and (3) except

* Presented to the Society, February 23, 1935.

$\dagger$ For other proofs of the reciprocity law using this transformed criterion see a paper by Lange, Ein Elementarer Beweis des Reziprozitäts-gesetzes, Berichte der Koeniglichen Sachsischen Gesellschaft, vol. 48 (1896), p. 629; vol. 49 (1897), p. 607; see also P. Bachmann, Niedere Zahlentheorie, Part 1, 1902, pp. 256-261, and pp. 266-267. 
when $(q+1) / 2$ is even and $(p+q) / 2$ is odd, that is, when $p, q$ are each of the form $4 n+3$. In this case there is one odd remainder that does not belong to such a pair. If we denote by $a$ the number of odd remainders greater than $p$, it follows that $a$ is even if at least one of the two primes $p, q$ is of the form $4 n+1$, and odd if both are of the form $4 n+3$. Consequently

$$
a \equiv(p-1)(q-1) / 4 \quad(\bmod 2) .
$$

Now let $b$ denote the number of those odd remainders in (1) that are less than $p$. Then $(p / q)=(-1)^{a+b}$. Also, if $c$ denotes the number of least positive odd remainders in the series

$$
2 q, 4 q, 6 q, \cdots,(p-1) q(\bmod p),
$$

we have $(q / p)=(-1)^{c}$. Hence

$$
(p / q)(q / p)=(-1)^{a+b+c} \text {. }
$$

To complete the proof, we shall now show that the odd remainders in (1) that are less than $p$ are identical with the odd remainders in (5), and hence that $b=c$. Let

$$
2 \lambda p \equiv r \quad(\bmod q),
$$

where now $r$ is an odd remainder such that $0<r<p$, and $1 \leqq \lambda \leqq(q-1) / 2$. Hence

$$
2 \lambda p=(2 \mu-1) q+r,
$$

where $0<\mu<(p+1) / 2$. From this we obtain

$$
(p+1-2 \mu) q \equiv r \quad(\bmod p) .
$$

Conversely, from (8), where $1 \leqq \mu \leqq(p-1) / 2$, we obtain (7) with $0<\lambda<(q+1) / 2$.

Hence, as stated above, the odd remainders in (1) that are less than $p$ are identical with the odd remainders in (5), so that $b=c$. The theorem then follows from (4) and (6).

The University of Pennsylvania 